\title{
Therapeutic Effect of Bone-Filling Mesh Container in Treating Vertebral Metastases with Vertebral Body Posterior Marginal Damage
}

\author{
Zi-Kun Duan ${ }^{1}$, Xin-Guo Kang ${ }^{1}$, Jin-Feng Zou ${ }^{1}$, Sheng-Li Ye ${ }^{1}$ and Chun-Jing He ${ }^{1,{ }^{*}}$ \\ ${ }^{1}$ Department of Pain, People's Hospital of Guizhou Province, Guiyang, China \\ "Corresponding author: Department of Pain, People's Hospital of Guizhou Province, 550002, Guiyang, China. Email: hcj777330@163.com
}

Received 2018 November 15; Revised 2019 July 28; Accepted 2019 July 31.

\begin{abstract}
Background: In order to reduce the occurrence of bone cement leakage, bone filling mesh container technique can be a prior choice for the treatment of vertebral metastases with damaged posterior margin of the thoracolumbar vertebral body. Objectives: The purpose of this retrospective study was to compare the efficacy and safety of percutaneous balloon kyphoplasty (PKP) and bone filling mesh containers (BFMCS) in the treatment of vertebral metastases with posterior vertebral body damage. Patients and Methods: This is a retrospective study. From October 2016 to January 2018, 40 cases (72 vertebral bodies) of thoracolumbar osteolytic metastases were treated with vertebroplasty. Among them, 20 cases (37 vertebral bodies) were treated with PKP (PKP group), and 20 cases (35 vertebral bodies) were treated with BFMCS (BFMCS group). The operation time of the two groups was recorded, and visual analog scale (VAS), Oswestry disability index (ODI), intraoperative bone cement leakage and complications were observed before operation and 1 day, 1 month and 6 months after operation.

Results: All patients underwent successful operation. The operation time of the PKP group was $42.65 \pm 7.84$ minutes, and 42.95 \pm 8.48 minutes in the BFMCS group $(\mathrm{P}=0.91)$. Both groups differed significantly when the results were compared with those measured before treatment. VAS dropped from $7.50 \pm 0.95$ points before operation to $1.20 \pm 0.41$ points at 6 months follow up in PKP group $(\mathrm{P}<0.001)$, in the BFMCS group VAS dropped from $7.50 \pm 0.94$ points before operation to $1.45 \pm 0.51$ points at 6 months after operation $(\mathrm{P}<0.001)$. The ODI of the PKP group dropped from $75.80 \pm 4.76$ before operation to $12.05 \pm 1.47,6$ months after operation $(\mathrm{P}<0.001)$, ODI dropped from $75.00 \pm 4.34$ before operation to $11.60 \pm 1.39$ at 6 months follow up in the BFMCS group $(\mathrm{P}<$ 0.001). In the PKP group, 15 vertebral bodies $(40.5 \%, 15 / 37)$ occurred bone cement leakage, but the patients had no clinical symptoms of bone cement leakage. Cement leakage occurred in one case in the BFMCS group. There were no complications such as pulmonary embolism, paraplegia or perioperative death.

Conclusion: The application of bone-filling mesh container for treating patients with thoracolumbar osseointegrated metastases could significantly reduce the leakage rate of bone cement, and is similar to traditional PKP in pain relief and activity improvement.
\end{abstract}

Keywords: Vertebroplasty, Vertebral Metastases, Bone-Filling Mesh Container, Bone Cement Leakage

\section{Background}

The spine is a high-risk site for advanced tumor metastasis, and in $5 \%$ to $10 \%$ of patients with malignant tumors spinal metastases may occur (1), especially in the thoracolumbar vertebral body. Surgical treatment is often required when there is instability of the spine, severe pain or insensitivity to radiotherapy and chemotherapy (2). At present, minimally invasive surgery has become an important treatment for spinal metastases (3). Percutaneous vertebroplasty (PVP), as an important means of minimally invasive surgery, has been widely used in vertebral compression fractures caused by osteoporosis, vertebral hemangioma, multiple myeloma and vertebral metastases $(4,5)$. Bone cement leakage is the most common complication of
PVP, with an incidence of $22 \%$ to $82 \%$ (6). However, due to the rich blood supply to the vertebral body, the vertebral body and vertebral cortical bone destruction, the cement leakage rate in treating patients with vertebral osteolytic tumors is significantly higher than that in the treating osteoporotic vertebral fracture, and is as high as $37.5 \%$ in the treatment of vertebral body metastasis (7). Infiltration of cement into the spinal canal through the posterior wall of the incomplete vertebral body may lead to serious complications such as paraplegia.

\section{Objectives}

In order to reduce the occurrence of bone cement leakage, bone-filling mesh bags came into being and are used 
to compare the efficacy and safety of percutaneous balloon kyphoplasty (PKP) and bone filling mesh containers (BFMCS) in the treatment of vertebral metastases with posterior vertebral body damage. From October 2016 to January 2018, 40 patients with thoracolumbar osteolytic metastases were treated with percutaneous balloon kyphoplasty and bone-filling mesh bags in our department. The cement leakage rate and visual analog scale (VAS) score were evaluated. The disability index and other indicators were studied to compare the clinical efficacy of two surgical treatments for thoracolumbar osteolytic metastases.

\section{Patients and Methods}

This was a retrospective study. In clinical practice, these two surgical methods are used to treat vertebral metastases with vertebral body posterior marginal damage. Patients voluntarily chose the surgical method. We took a sample with simple random sampling methods from patients with the same type of surgery.

A total of 40 patients with thoracolumbar osteolytic metastases (72 vertebral bodies) who underwent vertebroplasty from October 2016 to January 2018 were enrolled, including 47 thoracic vertebrae and 25 lumbar vertebrae.

\subsection{General Information}

Inclusion criteria were as follows: (1) The whole body bone scan and CT or MRI examination confirmed the osteolytic changes of the thoracolumbar vertebrae in no more than three vertebral bodies, the lesion was confined to the vertebral body, and the pathological examination confirmed the metastatic tumor, (2) CT showed the posterior marginal cortical bone was damaged, (3) Patients had severe chest and back pain and no symptoms of neurological damage of the lower extremities. Exclusion criteria were as follows: vertebral compression fracture caused by simple osteoporosis, pathological results of vertebral body biopsy could not confirm the source of metastatic tumor, poor cardiopulmonary function and not tolerating surgery, disturbances of blood coagulation that were difficult to be corrected.

Twenty patients (37 vertebral bodies) were treated with PKP (PKP group). In the PKP group, there were 11 males and nine females with age ranging from 36 to 78 years (average, 61.8 years). Vertebral body distribution: T9, one vertebral body, T10, four vertebral bodies, T11, six vertebral bodies, T12, ten vertebral bodies, L1, eight vertebral bodies, L2, six vertebral bodies, L3, two vertebral bodies. Twenty patients (35 vertebral bodies) were treated with BFMCS (BFMCs group). In the BFMCs group, there were 12 males and eight females with age ranging from 38 to 80 years (average, 62.4years). Vertebral body distribution: T9, two vertebral bodies, T10, two vertebral bodies, T11, nine vertebral bodies, T12, eleven vertebral bodies, L1, six vertebral bodies, L2, four vertebral bodies, L3, one vertebral body. There were no significant differences in age, gender, and vertebral body distribution between the two groups. Primary tumors distribution showed 13 cases (32.5\%) of lung cancer, eight cases $(20.0 \%)$ of breast cancer, seven cases (17.5\%) of liver cancer, three cases (7.5\%) of kidney cancer, three cases $(7.5 \%)$ of thyroid cancer, two colorectal cancer patients (5.0\%), one case of prostate cancer (2.5\%), and three cases of other malignant tumors (7.5\%). Twenty cases had single vertebral lesions, 23 cases had two vertebral lesions, and two cases had three vertebral lesions.

\subsection{Surgical Methods}

All patients were placed in a prone position and routinely sterilized. The needle position was selected according to preoperative measurements, the position of the diseased vertebra was determined by X-ray fluoroscopy, and the anterioposterior and lateral film was obtained. After local anesthesia with $1 \%$ lidocaine, the puncture needle was used to puncture into to the posterior edge of the vertebral body through the pedicle, and the puncture needle was properly abducted and the needle tail was tilted to the head side. The needling direction and depth were continuously observed until achieving satisfactory location through monitoring the perspective of positive and lateral position. By combining the patient's basic disease with pathological examination, we could prove the source of the tumor. Each patient underwent pathological biopsy at the time of surgery. After obtaining the biopsy with a hollow drill, the solid vertebral body was drilled to the one third of the vertebral body, and the balloon was placed for expansion. In the PKP group, after the balloon was withdrawn, the polymethyl methacrylate bone cement was blended to the "drawing period", and the bone cement was injected under the C-arm fluoroscopy. In the BFMCS group, the balloon was taken out and a bone-filling mesh container was placed. The polymethyl methacrylate bone cement was blended to the "drawing period", and the bone cement was injected under the C-arm fluoroscopy with a spiral pressure pusher. The lateral fluoroscopy was used to examine the distribution of bone cement. After operation, the puncture needle was pulled out and partially covered with a sterile applicator. The patient's symptoms of lower limbs and respiratory discomfort were observed. Patients stayed in bed 6 hours after operation. 


\subsection{Observation Indicators and Assessment Methods}

The operation time was recorded for the two groups. Patient pain was assessed by VAS score as preoperative (T1), 1 day postoperative (T2), 1 month postoperative (T3), and 6 months postoperative (T4). Improvement of activities was evaluated according to Oswestry disability index (ODI) (5), including 10 activities such as face washing, weightlifting, walking, sleeping, sexual life, and social activities. Each part includes six options, representing 0 - 5 points. ODI = actual score $/ 50$ (maximum possible score) $\times$ $100 \%$. If there is a question that is not answered, the scoring method would be the actual score/45 (highest possible score) $\times 100 \%$. Patients were followed up in the clinic 1 and 6 months after discharge. X-ray examination was performed. If necessary, CT or MRI was performed. The pain VAS score and ODI were evaluated at each follow-up.

During the operation, the leakage of cement was observed under the C-arm fluoroscopy, the incidence of bone cement leakage and the leakage site (type I is the leakage of cement into the spinal canal, type II is leakage of cement into the paravertebral vein, type III is leakage of the cement into the paravertebral soft tissue, and type IV is leakage of the cement into the adjacent intervertebral disc), and the complications occurring during and after operation were recorded.

\subsection{Statistical Analysis}

Statistical analysis was performed using SPSS statistics for Windows version 17 (SPSS Inc., Chicago, Il. USA). Continuous and ordinal data were expressed as mean \pm standard deviation. Preoperative and postoperative ODI score were normally distributed and compared between different time sessions using repeated measure ANOVA, while VAS score were not normally distributed and compared between successive time sessions using Friedman test and Wilcoxon signed-rank test. In addition, comparison of ODI and VAS between the two groups in the same time sessions were accomplished by T-test and U-Mann Whitney tests. Leakage and its grade in two groups were compared using the chi-square test. P lower than 0.05 was considered as significant.

\section{Results}

The average operation time of the PKP group was 42.65 \pm 7.84 minutes, which was $42.95 \pm 8.48$ minutes in the BFMCS group. There was no significant difference between the two groups $(\mathrm{P}=0.91)$.

The VAS scores of the two groups were lower than those before surgery, and the difference was statistically significant $(\mathrm{P}<0.001)$. There was no significant difference in VAS scores between the two groups (Table 1). The Oswesty disability index before surgery was higher than that at all time points after surgery, and the difference was statistically significant $(\mathrm{P}<0.001)$. There was no significant difference in Oswesty disability index between the two groups at the same time point (Table 2 ).

In the PKP group, 15 (40.5\%, 15/37) vertebral bodies showed bone cement leakage to the intervertebral disc and paravertebral tissue, but no clinical symptoms were observed. Eleven of them were in the intervertebral space, three were in the paravertebral or paraspinal veins, and one leaked in the spinal canal. In the BFMCs group, only one vertebral body showed bone cement leakage to the paravertebral tissue, and the cement leakage rate was significantly lower than that in the PKP group, and the difference was statistically significant (Table 3 ).

There was no pulmonary embolism, paraplegia or perioperative death, and no complications such as pneumothorax, puncture site bleeding and wound infection.

\subsection{Typical Cases}

A 71-year-old man was hospitalized because of back pain and right lower limb pain for half a month. He was diagnosed with lung cancer and in his thoracic vertebrae tumor metastasis was found, and bone cement was injected while applying the bone-filling mesh container. The posterior wall of the incomplete vertebral body was filled with bone cement, and no bone cement leakage was observed. VAS scores before operation and at day 1, 1 month, and 6 months were 7, 2, 1, and 1 point, respectively. Oswestry disability scores before operation and at day 1, 1 month and 6 months were 81, 20, 9, and 8, respectively (Figure 1).

\section{Discussion}

The spine is a high-risk site for advanced tumor metastasis. The vertebral metastatic tumor commonly originates from lung cancer, breast cancer, gastrointestinal malignant tumor, prostate cancer, lymphoma and renal cancer (8). Thoracic and lumbar vertebrae are common sites of spinal metastases, with thoracic vertebrae being the most common (70\%), followed by lumbar vertebrae (20\%) and cervical vertebrae (10\%) (9). Vertebral metastases often invade the sclerotin to cause osteolytic destruction, resulting in vertebral pathological fractures, spinal instability, spinal cord and nerve root compression and other complications. These complications often require surgical treatment (2). Minimally invasive surgery has become an important treatment for spinal metastases (3). Percutaneous vertebroplasty (PVP) is an important tool for minimally invasive surgery and can be treated with PVP and PKP (10-13). 

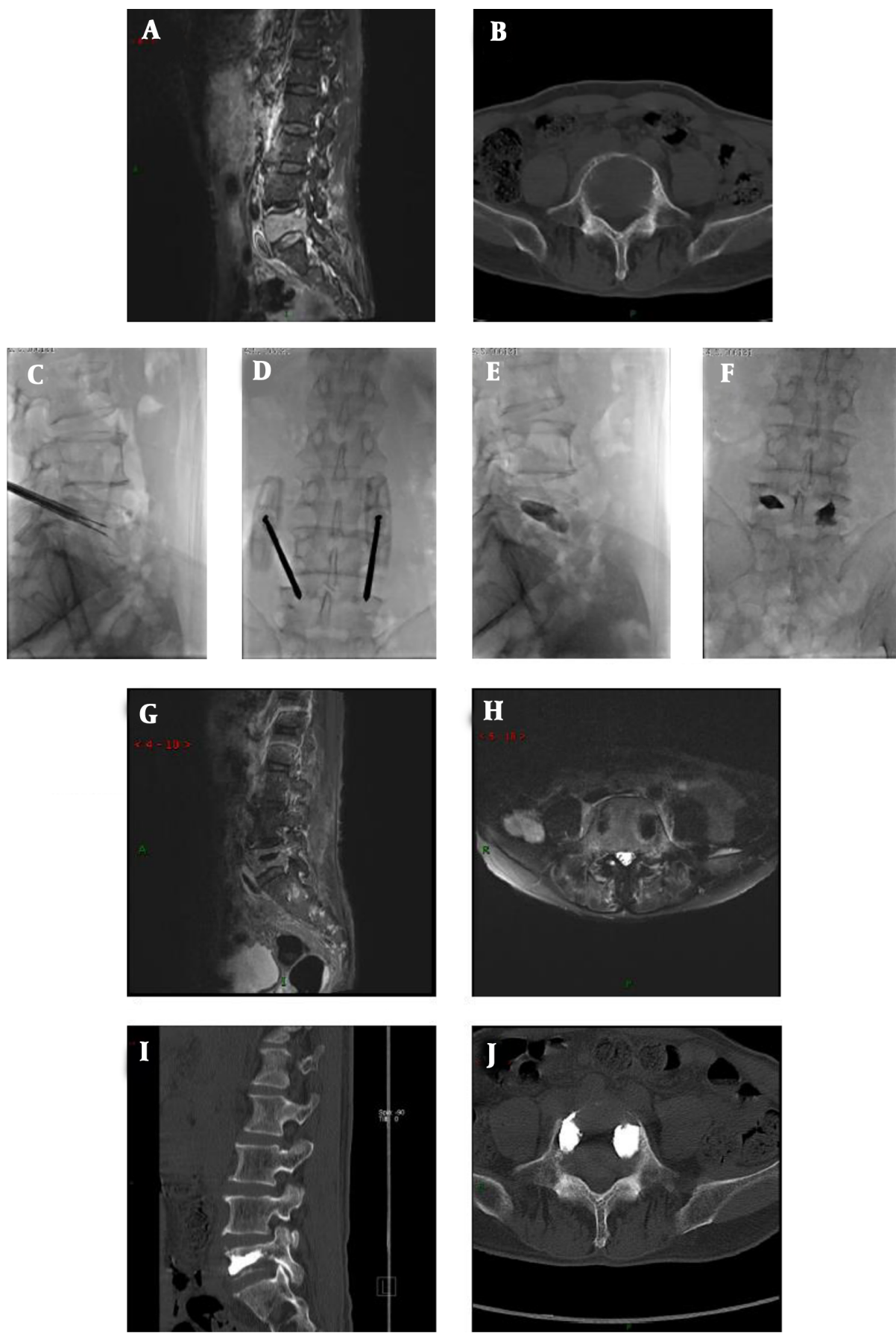

Figure 1. A 71-year-old man was hospitalized because of back pain and right lower limb pain for half month. He was diagnosed with lung cancer and in his thoracic vertebrae tumor metastasis was found. Visual analog scale (VAS), scores before operation and at day 1,1 month and 6 months were 7,2,1, and 1 points, respectively. Oswestry dysfunction scores before operation and at day 1,1 month and 6 months were 81, 20, 9, and 8, respectively. A and B, Before operation, MRI showed T5 vertebral slight wedge, a long T2 signal change, obscure boundary. CT showed a T5 vertebral osteolytic damage, soft tissue density shadow in vertebral body, and obscure boundary. C-F, During operation, the vertebral body was punctured through the bilateral vertebral pedicle approach, the mesh bag implanted, and the bone cement filled well without leakage. G-J, After operation, MRI showed T5 vertebral was filled with bone cement, both sides of vertebra with strip short signal change. CT showed both sides of T5 vertebral with high density shadow, and bone cement filled well without leakage. 


\begin{tabular}{lcccc}
\hline Table 1. Comparison of Preoperative and Postoperative VAS Scores Between the Two Groups & \\
\hline Groups & T1 & T2 & T3 & T41 \\
\hline PKP group & $7.50 \pm 0.95$ & $2.30 \pm 0.66^{\mathrm{c}}$ & $1.60 \pm 0.50^{\mathrm{c}}$ \\
BFMCS group & $7.50 \pm 0.94$ & $2.35 \pm 0.59^{\mathrm{c}}$ & $1.20 \pm 0.41^{\mathrm{c}} \pm 0.51^{\mathrm{c}}$ \\
P value & 0.73 & 0.77 & 0.75 & $1.45 \pm 0.51^{\mathrm{c}}$ \\
\hline
\end{tabular}

Abbreviations: BFMCS, bone filling mesh containers; PKP, percutaneous balloon kyphoplasty; SD, standard deviation; VAS, visual analog scale

${ }^{a}$ Values are expressed as mean \pm SD.

${ }^{\mathrm{b}} \mathrm{T} 1=$ before operation; $\mathrm{T} 2=1$ day after operation; $\mathrm{T} 3=1$ month after operation, $\mathrm{T} 4=6$ months after operation.

${ }^{\mathrm{C}}$ Statistical significance in comparison with $\mathrm{T} 1$.

\begin{tabular}{lcccc}
\hline \multicolumn{2}{l}{ Table 2. Comparison of Preoperative and Postoperative ODI Values Between the Two Groups } & \\
\hline Groups & T1 & T2 & T3 & T41 \\
\hline PKP group & $75.80 \pm 4.76$ & $26.25 \pm 2.86^{\mathrm{c}}$ & $16.85 \pm 1.66^{\mathrm{c}}$ \\
BFMCS group & $75.00 \pm 4.34$ & $26.35 \pm 2.78^{\mathrm{c}}$ & $16.10 \pm 1.62^{\mathrm{c}}$ & $1.05 \pm 1.47^{\mathrm{c}}$ \\
t value & 0.56 & -0.11 & 0.99 \\
P value & 0.58 & 0.91 & 0.16 \\
\hline
\end{tabular}

Abbreviations: BFMCS, bone filling mesh containers; PKP, percutaneous balloon kyphoplasty; SD, standard deviation; ODI, Oswestry disability index

${ }^{\mathrm{a}}$ Values are expressed as mean $\pm \mathrm{SD}$.

${ }^{\mathrm{b}} \mathrm{T} 1=$ before operation; $\mathrm{T} 2=1$ day after operation; $\mathrm{T} 3=1$ month after operation, $\mathrm{T} 4=6$ months after operation

${ }^{\mathrm{c}}$ Statistical significance in comparison with $\mathrm{T} 1$.

\begin{tabular}{lcccccc}
\hline Table 3. Bone Cement Leakage in Both Groups & & & & \\
\hline Group & I & II & III & V & Percentage of bone cement leakage \\
\hline PKP group & 1 & 3 & 11 & 0 & $40.5 \%$ & $2.9 \%^{\mathrm{a}}$ \\
BFMCS group & 1 & 0 & 0 & 0 & 51.00 & $<0.001$ \\
$\chi^{2}$ value & & & & & & \\
P value & & & & & \\
\hline
\end{tabular}

Abbreviations: BFMCS, bone filling mesh containers, PKP, percutaneous balloon kyphoplasty

${ }^{\mathrm{a}}$ Statistical significance in comparison with PKP.

PVP was first used in clinical practice in 1985. Because of its advantages such as significant pain relief, short time, less bleeding, and quick recovery, PVP has been widely used and achieved good results (14-22). The most common complication of PVP is bone cement leakage, and its incidence is up to $11 \%-76 \%(23,24)$. It most often occurs in vertebral bone damage or weakness $(25,26)$. The leakage rate of spinal metastases is often higher than that of osteoporotic vertebral fractures, which may be caused because tumor destructs cortical bone of the vertebral body or that tumor richens blood vessels and blood supply (27). Percutaneous balloon dilatation vertebral kyphoplasty is improved based on PVP, which can reduce the leakage rate to $8.4 \%$ (28-31). In order to further reduce the leakage of bone cement, bone filling mesh bags were made.

In this study, the VAS scores of the two groups were lower than those before surgery, and the difference was statistically significant. There was no significant difference in VAS scores between the two groups. The Oswesty disability index at all time points after surgery was lower than that before operation, and the difference was statistically significant. There was no significant difference in Oswesty disability index between the two groups at the same time point. It shows that the use of bone filling mesh bags and simple percutaneous balloon dilatation vertebroplasty have good curative effects, which can effectively relieve pain and improve motor.

In this study, bone cement leakage was observed in only one patient in the bone filling mesh container vertebroplasty group, and the leakage rate was significantly lower than that in the PKP group. The mesh container used in the research is a newly developed domestic expandable mesh bag-shaped bone material filler, which is intertwined into a mesh tubular structure by polyethylene terephthalate (PET), so as to utilize the wrapping role of the net container to reduce the leakage of bone cement caused by traditional PVP. After the bone filling mesh container is placed in the vertebral body, the bone cement is directly 
injected into the mesh bag, and the bone cement causes the mesh container structure to slowly expand. Similar to the PKP balloon expansion, it could raise partial height of the vertebral body, improve the stability of the spine, so as to achieve the purpose of relieving pain; meanwhile, it could also make the bone cement extend into the trabecular bone gap to form a micro-locking, which hinders the further exudation of the bone cement, thereby reducing the leakage rate of the bone cement. Its mechanical action also blocks the blood supply of the tumor, allowing the tumor to form ischemic or congestive necrosis. When the bone cement reaches a temperature of $70^{\circ} \mathrm{C}$ during polymerization, it could directly kill the tumor, and the cytotoxicity of the monomer could also kill the tumor.

In summary, the bone filling mesh container group achieves satisfactory clinical results in terms of postoperative pain improvement, and greatly reduces the incidence of intraoperative cement leakage. Therefore, considering of the effectiveness and safety of the patient's surgery, bone filling mesh container technique could be a prior choice for the treatment of vertebral metastases with damaged posterior margin of the thoracolumbar vertebral body. However, the application time of bone filling mesh container technology in clinical practice is short, the related reports are few, the long-term follow-up data is insufficient, and it is unclear whether the dispersion and distribution of bone cement are limited. Therefore, largesample, multi-center, and long-term follow-up studies are needed.

\section{Acknowledgments}

The authors would like to thank the nurses, volunteers, and patients who participated. The authors declare no conflicts of interest.

\section{Footnotes}

Authors' Contributions: Study concept and design: Chun-Jing He MD, drafting of the manuscript and critical revision of the manuscript for important intellectual content: Zi-Kun Duan, analysis and interpretation of data: XinGuo Kang and Jin-Feng Zou, statistical analysis: Sheng-Li Ye. Xin-Guo Kang should be regarded as co-first authors.

Conflict of Interests: It is not declared by the authors.

Ethical Approval: This study was approved by the institutional review board in the hospital.

Financial Disclosure: It is not declared by the authors.

Funding/Support: It is not declared by the authors.

Patient Consent: Written and signed informed consent was obtained from all participants prior to examination.

\section{References}

1. Yang Z, Xu Y, Yang D, Sun H, Zhao R, Zhang J, et al. Pathological impairments induced by interstitial implantation of $125 \mathrm{I}$ seeds in spinal canal of banna mini-pigs. World J Surg Oncol. 2012;10:48 doi: 10.1186/1477-7819-10-48. [PubMed: 22390740]. [PubMed Central: PMC3312839].

2. Langdon J, Way A, Heaton S, Bernard J, Molloy S. The management of spinal metastases from renal cell carcinoma. Ann $R$ Coll Surg Engl. 2009;91(8):649-52. doi: 10.1308/003588409X432482. [PubMed: 19686617]. [PubMed Central: PMC2966239].

3. Harel R, Angelov L. Spine metastases: Current treatments and future directions. Eur J Cancer. 2010;46(15):2696-707. doi: 10.1016/j.ejca.2010.04.025. [PubMed: 20627705].

4. Bozkurt M, Kahilogullari G, Ozdemir M, Ozgural O, Attar A, Caglar S, et al. Comparative analysis of vertebroplasty and kyphoplasty for osteoporotic vertebral compression fractures. Asian Spine J.2014;8(1):2734. doi: 10.4184/asj.2014.8.1.27. [PubMed: 24596602]. [PubMed Central: PMC3939366].

5. Chew C, Ritchie M, O'Dwyer PJ, Edwards R. A prospective study of percutaneous vertebroplasty in patients with myeloma and spinal metastases. Clin Radiol. 2011;66(12):1193-6. doi: 10.1016/j.crad.2011.08.004. [PubMed: 21968026].

6. Martin DJ, Rad AE, Kallmes DF. Prevalence of extravertebral cement leakage after vertebroplasty: Procedural documentation versus CT detection. Acta Radiol. 2012;53(5):569-72. doi: 10.1258/ar.2012.120222. [PubMed: 22637642].

7. Nieuwenhuijse MJ, Van Erkel AR, Dijkstra PD. Cement leakage in percutaneous vertebroplasty for osteoporotic vertebral compression fractures: Identification of risk factors. Spine J. 2011;11(9):839-48. doi: 10.1016/j.spinee.2011.07.027. [PubMed: 21889417].

8. Sioutos PJ, Arbit E, Meshulam CF, Galicich JH. Spinal metastases from solid tumors. Analysis of factors affecting survival. Cancer. 1995;76(8):1453-9. doi: 10.1002/1097-0142(19951015)76:8<1453::aidcncr2820760824>3.0.co;2-t. [PubMed: 8620423].

9. Klimo PJr, Schmidt MH. Surgical management of spinal metastases. Oncologist. 2004;9(2):188-96. doi: 10.1634/theoncologist.9-2-188. [PubMed: 15047923].

10. Wood KB, Li W, Lebl DR, Ploumis A. Management of thoracolumbar spine fractures. Spine J. 2014;14(1):145-64. doi: 10.1016/j.spinee.2012.10.041. [PubMed: 24332321].

11. Teyssedou S, Saget M, Pries P. Kyphopasty and vertebroplasty. Orthop Traumatol Surg Res. 2014;100(1 Suppl):S169-79. doi: 10.1016/j.otsr.2013.11.005. [PubMed: 24406028].

12. Voormolen MH, Mali WP, Lohle PN, Fransen H, Lampmann LE, van der Graaf Y, et al. Percutaneous vertebroplasty compared with optimal pain medication treatment: short-term clinical outcome of patients with subacute or chronic painful osteoporotic vertebral compression fractures. The VERTOS study. AJNR Am J Neuroradiol. 2007;28(3):555-60. [PubMed: 17353335].

13. Han S, Park HS, Pee YH, Oh SH, Jang IT. The clinical characteristics of lower lumbar osteoporotic compression fractures treated by percutaneous vertebroplasty: A comparative analysis of 120 cases. Korean J Spine. 2013;10(4):221-6. doi: 10.14245/kjs.2013.10.4.221. [PubMed: 24891852]. [PubMed Central: PMC4040645].

14. Galibert P, Deramond H, Rosat P, Le Gars D. [Preliminary note on the treatment of vertebral angioma by percutaneous acrylic vertebroplasty]. Neurochirurgie. 1987;33(2):166-8. French. [PubMed: 3600949].

15. Sun H, Yang Z, Xu Y, Liu X, Zhang Y, Chen Y, et al. Safety of percutaneous vertebroplasty for the treatment of metastatic spinal tumors in patients with posterior wall defects. Eur Spine J. 2015;24(8):1768-77. doi: 10.1007/s00586-015-3810-8. [PubMed: 25694161]. 
16. Shimony JS, Gilula LA, Zeller AJ, Brown DB. Percutaneous vertebroplasty for malignant compression fractures with epidural involvement. Radiology. 2004;232(3):846-53. doi: 10.1148/radiol.2323030353. [PubMed: 15273339].

17. Sun G, Li L, Jin P, Liu XW, Li M. Percutaneous vertebroplasty for painful spinal metastasis with epidural encroachment. J Surg Oncol. 2014;110(2):123-8. doi: 10.1002/jso.23608. [PubMed: 24665071].

18. Chew C, Craig L, Edwards R, Moss J, O’Dwyer PJ. Safety and efficacy of percutaneous vertebroplasty in malignancy: A systematic review. Clin Radiol. 2011;66(1):63-72. doi: 10.1016/j.crad.2010.09.011. [PubMed: 21147301].

19. Trumm CG, Pahl A, Helmberger TK, Jakobs TF, Zech CJ, Stahl R, et al. CT fluoroscopy-guided percutaneous vertebroplasty in spinal malignancy: Technical results, PMMA leakages, and complications in 202 patients. Skeletal Radiol. 2012;41(11):1391-400. doi:10.1007/s00256-0121365-x. [PubMed: 22286549].

20. Tancioni F, Lorenzetti MA, Navarria P, Pessina F, Draghi R, Pedrazzoli $\mathrm{P}$, et al. Percutaneous vertebral augmentation in metastatic disease: State of the art. J Support Oncol. 2011;9(1):4-10. doi: 10.1016/j.suponc.2011.01.001. [PubMed: 21465731].

21. Goldstein CL, Chutkan NB, Choma TJ, Orr RD. Management of the elderly with vertebral compression fractures. Neurosurgery. 2015;77 Suppl 4:S33-45. doi: 10.1227/NEU.0000000000000947. [PubMed: 26378356].

22. Du J, Li X, Lin X. Kyphoplasty versus vertebroplasty in the treatment of painful osteoporotic vertebral compression fractures: twoyear follow-up in a prospective controlled study. Acta Orthop Belg. 2014;80(4):477-86. [PubMed: 26280719].

23. Nieuwenhuijse MJ, van Erkel AR, Dijkstra PD. Percutaneous vertebroplasty in very severe osteoporotic vertebral compression fractures: Feasible and beneficial. J Vasc Interv Radiol. 2011;22(7):1017-23. doi: 10.1016/j.jvir.2011.02.036. [PubMed: 21571548]
24. O'Brien JP, Sims JT, Evans AJ. Vertebroplasty in patients with severe vertebral compression fractures: A technical report. AJNR Am J Neuroradiol. 2000;21(8):1555-8. [PubMed: 11003296].

25. Hulme PA, Krebs J, Ferguson SJ, Berlemann U. Vertebroplasty and kyphoplasty: A systematic review of 69 clinical studies. Spine (Phila Pa 1976). 2006;31(17):1983-2001. doi: 10.1097/01.brs.0000229254.89952.6b. [PubMed: 16924218].

26. Muijs SP, Nieuwenhuijse MJ, Van Erkel AR, Dijkstra PD. Percutaneous vertebroplasty for the treatment of osteoporotic vertebral compression fractures: Evaluation after 36 months.J Bone Joint Surg Br. 2009;91(3):379-84. doi: 10.1302/0301-620X.91B3.20970. [PubMed: 19258616].

27. Jensen ME, Kallmes DE. Percutaneous vertebroplasty in the treatment of malignant spine disease. Cancer J. 2002;8(2):194-206. doi: 10.1097/00130404-200203000-00013. [PubMed: 11999951].

28. Peh WC, Gilula LA, Peck DD. Percutaneous vertebroplasty for severe osteoporotic vertebral body compression fractures. Radiology. 2002;223(1):121-6. doi: 10.1148/radiol.2231010234. [PubMed: 11930056].

29. Young C, Munk PL, Heran MK, Lane MD, Le HB, Lee S, et al. Treatment of severe vertebral body compression fractures with percutaneous vertebroplasty. Skeletal Radiol.2011;40(12):1531-6. doi:10.1007/s00256-0111138-y. [PubMed: 21442418].

30. Pflugmacher R, Beth P, Schroeder RJ, Schaser KD, Melcher I. Balloon kyphoplasty for the treatment of pathological fractures in the thoracic and lumbar spine caused by metastasis: One-year followup. Acta Radiol. 2007;48(1):89-95. doi: 10.1080/02841850601026427. [PubMed: 17325932].

31. He CJ, Liu GD. Comparison of the efficacy and safety of bone-filling mesh container and simple percutaneous balloon kyphoplasty in the treatment of osteoporotic vertebral compression fractures. Pain Physician. 2018;21(3):259-68. [PubMed: 29871370]. 